\title{
Research dynamics depends on a journal publication.
}

\author{
Asmita Sengupta* \\ Professor of Physics, Department of Physics, Visva-Bharati University, Santiniketan, Bolpur, West Bengal, India
}

Accepted on September 13, 2017

\section{Editorial}

This is interesting, but true. As a subject, chemistry is young but the related technology is ancient. Humans have been using it but since the historians have forgotten to record, it has become impossible for us to tell you the exact date and year. Surely they were using various chemical processes even without having any idea of what they were doing but only knew that they were doing those inconsequential experiments to bring more comforts. To day even a kid knows detail about, say, curd, but imagine on those days when the dawn of civilization was not even at the horizon, this particular knowledge was invented by a person or a family following a chemical process even without knowing the forces used to get a complex food like curd! So chemical processes were there by virtue of accidents and some process was initiated as play but all those contributed to pick up a momentum in developing civilization.

The history of progress of chemistry and chemical processes taught us that in the past progress was slow, uneven and inadequate because there was practice but no theory. But the union of theory with practice brought a paradigm shift in the progress of Chemistry, Chemical technology, engineering, process science, related educational curriculum and research. All these in a synthesized fashion contribute to the progress of human civilization. Name of material which has neither past nor future without intervention of chemical technology and applications, now-a-days has emerged with a number of sub topics. Now this industry is worth almost 3 trillion Dollars globally and thus this vast industry is compelled to incorporate disciplines of pure science and engineering. New syllabus and curriculum have been introduced in leading universities and institutions to support research and service for this sector. Research on chemical technology aims for breakthroughs which may lead to the discovery of new materials having tangible and physical presence. When a researcher will announce the discovery of a novel substance, most probably we will be the first to appreciate that research and present our Editorial team's assessment of the impact of those discoveries on society. We are curious to know, through your research submissions, about the great diversity in materials research, irrespective of whether in science or engineering. We invite all researchers to deal with issues related to cars, machines, electrical devices, textiles, medicine, and energy etc. This journal encourages submissions on research knowledge of chemical reactions, material synthesis, function design, material transformations, production technology etc. We are certainly interested to achieve a greener society through advanced engineering research in the chemical industry.

The journal attempts to participate in this greatest human effort to standardize and include more materials for material dependent human life and to survive by publishing original, comprehensive and new models of theory and measurement protocols. The journal editorial staffs are very active and smart in introducing a focused, quick review system for fastest publication. Why so? Simply, we understand from our research day experiences that a researcher of any level and position wants to see 'a decision' on work as quickly as possible. Publishing, a process to strengthen only their scholastic mission. Long way to go for this subject and we stand by in this quest. You may join us!

\section{*Correspondence to:}

Asmita Sengupta
Department of Physics,
Visva-Bharati University
Public University in Santiniketan,
Bolpur, West Bengal
India
Tel: 91-9434589622
E-mail: asmita_sengupta@hotmail.com

